\title{
ON ALMOST PERIODIC DIFFERENTIAL EQUATIONS
}

\author{
BY RICHARD K. MILLER ${ }^{1}$
}

\author{
Communicated by H. A. Antosiewicz, July 13, 1964
}

The notion of an invariant set has been widely exploited in the theory of autonomous ordinary differential equations. The purpose of this announcement is to give a natural and useful generalization of invariant sets to almost periodic systems. The proofs and several applications and extensions of our results will appear elsewhere.

We consider systems of $n$ first order, almost periodic, ordinary differential equations of the form

$$
x^{\prime}=P(t, x) \quad\left(\prime=\frac{d}{d t}\right),
$$

and perturbations of the form

$$
x^{\prime}=P(t, x)+R(t, x)+G(t, x) .
$$

The main result of this paper is Theorem 1 below. This rather abstract-looking theorem motivates our generalization of invariant sets. Moreover, Theorem 1 has applications to perturbation theory and to the theory of Liapunov functions. In fact, several known results for asymptotic behavior of solutions of nonlinear systems may be proved using this result (cf. [6], [7], [8], [10], and [5, p. 69]).

Let $D$ be a fixed open domain in $n$-space $R^{n}$. Let $Q$ be a fixed subset of $D$ which is closed in the topology of $D$. We assume that the functions $P, R$, and $G$ of systems (E) and (PE) satisfy the following hypotheses.

(H1) $R$ and $G$ are continuous on $I \times D, I=\{t ; 0 \leqq t<\infty\}$.

(H2) $P$ is continuous on $R^{1} \times D$ and for each compact subset $D^{*}$ of $D, P$ is uniformly continuous on $R^{1} \times D^{*}$.

(H3) For each fixed $x$ in $D, P(t, x)$ is almost periodic as a function of $t$ in the sense of H. Bohr, cf. [4].

(H4) If $y(t)$ is any continuous function on the interval $I$ with values in a compact set $D^{*} \subset D$, then $|G(t, y(t))| \in L_{1}(0, \infty)$, where $|G(t, y(t))|$ is the vector norm of $G(t ; y(t))$.

(H5) $R(t, x) \rightarrow 0$ as $t \rightarrow \infty$ uniformly for $x$ on compact subsets of $Q$.

(H6) For each $\epsilon>0$ and each $x$ in $Q$ there are numbers $T>0$ and $\delta>0$ such that whenever $t \geqq T$ and $|x-y|<\delta$ one has

\footnotetext{
${ }^{1}$ Partially supported by the National Science Foundation (G-24335).
} 


$$
|R(t, x)-R(t, y)|<\epsilon \text {. }
$$

If $P(t, x)$ satisfies $(\mathrm{H} 2)$ and $(\mathrm{H} 3)$, we shall call $P(t, x)$ almost periodic in $t$. If $P(t, x)$ is almost periodic in $t$, then the hull of $P$ is the set of functions $\{P(t+h, x) ;-\infty<h<\infty\}$. The closed hull of $P$, denoted by $\bar{H}(P)$, is the uniform closure (over all $t$ and over $x$ on compact subsets of $D$ ) of $H(P)$. Using the set $\bar{H}(P)$, our main result may be stated as follows:

TheOREm 1. Let $x(t)$ be a solution of system (PE) defined on an interval $t_{0} \leqq t<\infty$ with values in a compact subset $D^{*}$ of $D$. If $x(t) \rightarrow Q$ as $t \rightarrow \infty$, then to each point $z$ in the positive limit set of $x(t)$ there corresponds a sequence $t_{m} \rightarrow \infty$ as $m \rightarrow \infty$, a function $P^{*}(t, x) \in \bar{H}(P)$ and a function $y(t)$ such that

(a) $y^{\prime}(t)=P^{*}(t, y(t))$ for $-\infty<t<\infty$ and $y(0)=z$,

(b) $x\left(t+t_{m}\right) \rightarrow y(t)$ as $m \rightarrow \infty$ uniformly on compact subsets of $-\infty<t<\infty$, and

(c) $P\left(t+t_{m}, x\right) \rightarrow P^{*}(t, x)$ as $m \rightarrow \infty$ uniformly for all $t$ and $x$ on compact subsets of $D$.

The conclusion of Theorem 1 suggests the following generalization of invariance for system (E): If $A \subset D$, then $A$ is called quasi-invariant with respect to the system $(\mathrm{E})$ if for each point $z \in A$ there exists a $P^{*} \in \bar{H}(P)$ and a solution $y(t)$ of the system

$$
y^{\prime}=P^{*}(t, y)
$$

with $y(0)=z$, such that $y(t)$ exists as a solution of $\left(\mathrm{E}^{*}\right)$ and remains in a compact subset of $A$ for $-\infty<t<\infty$.

The content of Theorem 1 is that the positive limit set of the solution $x(t)$ is quasi-invariant with respect to system (E). Since the bounded solution $x(t)$ must approach its positive limit set as $t \rightarrow \infty$, we have immediately:

Corollary 1. Let the hypotheses of Theorem 1 hold. Let $Q_{0}$ be the largest quasi-invariant subset of $Q$ with respect to (E). Then $x(t) \rightarrow Q_{0}$ as $t \rightarrow \infty$.

Since the definition of quasi-invariance includes the notion of boundedness, we also conclude the following:

Corollary 2. Suppose there is a $P^{*} \in \bar{H}(P)$ such that no solution of $y^{\prime}=P^{*}(t, y)$ remains in a compact subset $D^{*} \subset D$ for $-\infty<t<\infty$. Then no solution $x(t)$ of (PE) which is bounded in a compact subset of $D$ can approach $Q$ as $t \rightarrow \infty$. 
We remark that Yoshizawa [10] has proved Theorem 1 in the special case $P(t, x)=P(x)$ is independent of $t$. We note that in case $Q=D$ and $R(t, x) \equiv G(t, x) \equiv 0$, (PE) reduces to (E) and satisfies hypotheses (H1)-(H6). In this special case, Corollary 2 reduces to a result of Amerio [1, pp. 97-105]. Corollary 1 together with certain results of Yoshizawa [10, pp. 382 and 385] may be used to generalize some results of Levin [7].

LaSalle [6] obtains results on the asymptotic behavior of autonomous and periodic systems of differential equations by combining the method of Liapunov with the notion of an invariant set. Using Theorem 1 above, LaSalle's result may be generalized as follows:

Theorem 2. Let $P(t, x)$ be almost periodic in $t$. Suppose there is a function $V(t, x)$ such that

(i) $V(t, x)$ is locally lipschitzian in $x$ and almost periodic in $t$, and

(ii) the derivative $\dot{V}(t, x)$, defined by

$$
V(t, x)=\limsup _{h \rightarrow 0^{+}} \frac{1}{h}(V(t+h, x+h P(t, x))-V(t, x)),
$$

is a nonpositive function which is almost periodic in $t$.

Let

$$
A=\left\{x \in D ; \inf _{-\infty<t<\infty}-\dot{V}(t, x)=0\right\}
$$

and let $A_{0}$ denote the largest quasi-invariant subset of $A$ with respect to (E). Then all bounded solutions of (E) approach $A_{0}$ as $t \rightarrow \infty$.

Corollary 3. If in Theorem 2 we have $V(t, x)$ positive definite and $A_{0}$ equal to the origin, then $x \equiv 0$ is an asymptotically stable solution of $(\mathrm{E})$.

In view of Theorem 2 it would be interesting to know when system (E) admits a Liapunov function $V(t, x)$ which is almost periodic in $t$. The following result provides a partial answer to this question.

Theorem 3. Suppose $P(t, x)$ is almost periodic in $t$ and is lipschitzian in $x$ (uniformly in $t$ ). If $x \equiv 0$ is a uniform-asymptotically stable solution of system (E), then there is a function $V(t, x)$ satisfying the following conditions:

(i) $V(t, x)$ is lipschitzian in $x$ (uniformly in $t$ ) and is almost periodic in $t$,

(ii) $V(t, x) \rightarrow 0$ as $|x| \rightarrow 0$ uniformly for $t \in R^{1}$,

(iii) $V(t, x)$ is positive definite, and 
(iv) the derivative $\dot{V}(t, x)$ with respect to system (E) is negative definite and almost periodic in $t$.

The proof of Theorem 3 is similar to the proof of a converse theorem given by Massera, cf. [9] or [2, pp. 155-157]. The function $V(t, x)$ has the form

$$
V(t, x)=\int_{0}^{\infty} G(|F(t+s, t, x)|) d s,
$$

where $F\left(t, t_{0}, x_{0}\right)$ is the solution of (E) through the initial point $\left(t_{0}, x_{0}\right)$ and the function $G(s)$ is chosen using Massera's lemma, cf. [9] or $[2$, p. 145$]$.

\section{BIBLIOGRAPHY}

1. L. Amerio, Soluzioni quasi-periodiche, o limitati, di sistemi differenziali non lineari quasi-periodici, o limitati, Ann. Mat. Pura Appl. 39 (1955), 97-119.

2. H. A. Antosiewicz, A survey of Lyapunov's second method, Contributions to the theory of nonlinear oscillations, Vol. 4, pp. 141-166, Annals of Mathematics Studies No. 41, Princeton Univ. Press, Princeton, N. J., 1958.

3. E. A. Barbašin and N. N. KrasovskiY, On stability of motion in the large, Dokl. Akad. Nauk SSSR 86 (1952), 453-456.

4. A. S. Besicovitch, Almost periodic functions, Dover, New York, 1954.

5. N. N. Krasovski1, Stability of motions, Stanford Univ. Press, Stanford, Calif., 1963.

6. J. LaSalle, Asymptotic stability criterion, Proc. Sympos. Appl. Math. Vol. 13, pp. 299-307, Amer. Math. Soc., Providence, R. I., 1962.

7. J. J. Levin, On the global asymptotic behavior of nonlinear systems of differential equations, Arch. Rational Mech. Anal. 6 (1960), 65-74.

8. J. J. Levin and J. A. Nohel, Global asymptotic stability of nonlinear systems of differential equations and applications to reactor dynamics, Arch. Rational Mech. Anal. 5 (1960), 194-211.

9. J. L. Massera, On Liapounoff's conditions of stability, Ann. of Math. (2) 50 (1949), 705-721.

10. T. Yoshizawa, Asymptotic behavior of solutions of a system of differential equations, Contributions to Differential Equations 1 (1963), 371-387.

11. - A Asymptotic system of a perturbed system, Internat. Sympos. Nonlinear Differential Equations and Nonlinear Mechanics, pp. 80-85, Academic Press, New York, 1963.

UNIVERSITY OF WiSCONSIN 